\title{
Surface potentials and electric dipole moments of ganglioside and phospholipid monolayers: contribution of the polar headgroup at the water/lipid interface
}

\author{
Heinz Beitinger ' , Viola Vogel ${ }^{2}$, Dietmar Möbius ${ }^{2}$ and Hinrich Rahmann ${ }^{1}$ \\ 'Institut für Zoologie, Universität Stuttgart-Hohenheim, Stuttgart and 'Max-Planck-Institu! für bicphysikalische Chemie. \\ Görtingen-Nikolausberg (F.R.G.)
}

(Received 20 January 1989)

Key words: Ganglioside; Phospholipid; Monolayer: Water/lipid interface: Lipid/air interface: Surface potential: Electric dipole moment

Monolayers of different gangliosides $\left(G_{M 1}, G_{D l a}, G_{D 1 b}, G_{T \text { Tlb }}, G_{\text {Mix }}\right.$ ), ceramide (Cer), sulfatide (Sulf), phospholipids (DOPC, DPPE, DPPS, DOPA), a quaternary ammonium salt (DOMA) and fatty acids $\left(C_{16}, C_{18}, C_{20}\right.$ ) were investigated at the air/water interface on pure water as well as on buffered subphases. Monolayers at the air/water interface consist of two interfaces: the water/lipid and the lipid/air interfaces. The normal components of the effective total dipole moments $\left(\Delta_{\perp} \mu\right)$, the effective local dipole moments $\left(\Delta_{\perp} \mu_{\alpha}\right)$ and effective local surface potentials $\left(\Delta V_{a}\right)$ of polar headgroups at the water/lipid interface have been calculated from surface potential $(\Delta V)$ and mean molecular area $(A)$ measurements of close-packed monolayers. The contribution of the lipid/air interface was previously determined experimentally by partial dipole compensation approach (Vogel, V. and Möbius, D. (1988) Thin Solid Films 159, 73-81). The surface potentials $(\Delta V)$ of ganglioside monolayers are quite similar (e.g., $G_{M 1}=-17 \mathrm{mV}$, $G_{\text {Ttb }}=-39 \mathrm{mV}$; at surface pressure $\pi=30 \mathrm{mN} / \mathrm{m}$, on $5 \mathrm{mM}$ triethanolamine (TEA)/HCl buffer, pH 7.4, as subphase); this indicates that variations in molecular structure of gangliosides like the influence of the number of negative charges per ganglioside which lead to appreciable changes in the average molecular packing do not cause large changes in surface potential. The local surface potentials $\left(\Delta V_{a}\right)$ reach to minus several hundred millivolts for nearly all compounds, but clear differences are shown between negatively charged phospholipids (e.g., DPPS $=-296 \mathrm{mV}$ at $\pi=30 \mathrm{mN} / \mathrm{m}$ ) and glycolipids (e.g., $G_{M 1}=-413 \mathrm{mV}$ ), and within glycolipids at different surface pressures (e.g. $G_{\text {p1: }}:-342 \mathrm{mV}$ at $\pi=20 \mathrm{mN} / \mathrm{m}$ versus $-453 \mathrm{mV}$ at $\pi=30 \mathrm{mN} / \mathrm{m}$ ). Only in gangliosides (except for $G_{\mathrm{Dlb}}$ ) the total dipole moments $\left(\Delta_{\perp} \mu\right)$ are negative $(-0.029$ up to $-0.078 \mathrm{D})$ and directed to the water. Unlike DOPC $(+0.069$ D) and DOMA $\left(+0.421\right.$ D), the local dipole moments $\left(\Delta_{,} \mu_{\alpha}\right)$ of all hydrated polar headgroups are negative (e.g., DPPS $\left.=-0.331 \mathrm{D} ; \mathrm{G}_{\mathrm{M} 1}=-0.729 \mathrm{D}\right)$ and directed from the monolayer $(-)$ to the water $(+)$. Under well-defined conditions investigated such data are helpful for a better understanding of the large functional role of gangliosides especially in determining the surface potential of biological membranes.

\section{Introduction}

Gangliosides are characteristic glycosphingolipids containing different numbers of negatively charged sialic acids. These molecules being particularly abundant in nerve cell membranes of vertebrates are components of the outer bilayer leaflet and might be intimately involved in various cellular biological events [1-3]. The functional role of gangliosides can be summarized under the following headings: cell-cell recognition and

Correspondence: H. Beitinger, Institut für Zoologie, Universität Stuttgart-Hohenheim, Garbenstrasse 30, D-7000 Stuttgart 70, F.R.G. adhesion, contact inhibition of cell growth, modulatory function for cell membrane receptors, membrane ion pumps and ion channels.

More than 2000 articles on gangliosides were published during the past decade, a lot of them with speciai regard to the large hydrophilic sugar moiety and its great potential for hydrogen-bonding. These reports give evidence that the polar headgroup determines the physicochemical properties of these molecules. Several studies $\left({ }^{13} \mathrm{C}\right.$-NMR, ESR, X-ray diffraction) on the orientation of the sugar headgroup indicate that the polar headgroup is relatively rigid, and in mixed phospholipid/ganglioside bilayers fully extended and approximately perpendicular to the interface [4-6]. 
For analyzing the physicochemical properties of gangliosides and different cell membrane molecules as well as their mutual interactions, investigations on monolayers as artificial and simple semimembrane systems might be suitable. The formation of a monolayer can be measured as a change in surface pressure and surface potential. The surface potential is proportional to the change of the normal component of the dipole density with regard to the pure water surface. Numerous reports exist of measurements with respect to the total surface potential and the overall dipole moment, respective!y, of simple glycosphingolipids and gangliosides [4,7-13]. In gangliosides, however, there are, in part, fundamental discrepancies in the surface pressure-area as well as in the surface potential-area isotherms obtained by the different groups, which will be discussed later.

In the present study especially the influence of the polar headgroup is investigated on the potential drop across the water/lipid interface that is created by the headgroup charges and their dipole moment. The formation of a lipid monolayer involves the creation of two interfaces, the water/lipid and the lipid/air interface. Attempts to calculate the dipole contribution per headgroup from surface potential data have been done earlier but only recently, the dipole contribution of the lipid/air interface of close-packed hydrocarbon chains was determined experimentally. The absolute dipole moment per terminal methyl group $\left(\mu^{\mathrm{CH}}\right)$ is $+0.35 \mathrm{D}$ [14]. Based on this constant value, the effective dipole contribution per headgroup at the water/lipid interface as well as the effective potential drop across that interface will be given for different gangliosides $\left(G_{M 1}, G_{D 1 a}\right.$, $G_{D 1 b}, G_{T l b}, G_{M i x}$ ), ceramide (Cer), sulfatide (Sulf), phospholipids (DOPC, DPPE, DPPS, DOPA), a quaternary ammonium salt (DOMA) and fatty acids $\left(C_{16}, C_{18}, C_{20}\right)$, on ion-free pure water as well as on buffered subphases.

The knowledge of these electrostatic data for hydrophilic interfaces is relevant for biological membrane systems since in biological bilayer membranes the dipole moments along the hydrophobic/hydrophobic contact line in the hydrophobic core are widely compensated and, therefore, the polar headgroups form the molecular basis for the electrical behaviour of the membrane surface.

\section{Materials and Methods}

Monolayers were formed at the air/water interface of a rectangular Teflon (polytetrafluorideethylene) trough (area, $355 \mathrm{~cm}^{2}$; depth, $1 \mathrm{~cm}$ ). The surface pressure was measured by a filter paper Wilhelmy balance, and the surface potentials were measured with a vibrating plate condensor. The principle of potential measurement has been described in a previous paper [14].
The surface pressure-area and surface potential-area isotherms have been determined for the following compounds: The monosialo- $\left(\mathrm{G}_{\mathrm{M} 1}\right)$, disialo- $\left(\mathrm{G}_{\mathrm{Dla}}\right)$, and trisialo-ganglioside $\left(\mathbf{G}_{\mathrm{T} 1 \mathrm{~b}}\right)$, ganglioside mixture $\left(\mathbf{G}_{\mathrm{Mix}}\right)$ from bovine brain (Fidia, Italy; Biosynth, Switzerland); disialo-ganglioside $\left(G_{\text {DIb }}\right)$ (Biosynth, Switzerland); sulfatide (Sulf), ceramide (Cer) (Sigma, F.R.G.); dioleoylphosphatidylcholine (DOPC), dipalmitoylphosphatidylethanolamine (DPPE), dipalmitoylphosphatidylserine (DPPS), dioleoylphosphatidic acid (DOPA) (Avanti Polar Lipids, Great Britain); dioctadecyldimethylammonium bromide (DOMA) (Sigma, F.R.G.); palmitic $\left(C_{16}\right)$, stearic $\left(C_{18}\right)$ and arachidic $\left(C_{20}\right)$ acid (Fluka, purified by repeated recrystallisation).

It is mentionable that most of the purified gangliosides used in this work were a kind gift from Fidia Research Labs., Abano Terme, Italy. Two of them, $G_{M 1}$ and $G_{\text {Mix }}$ ('Cronassial'), are drugs given in the therapy of neurological diseases. In control experiments purified gangliosides purchased from Biosynth AG, Staad, Switzeriand, showed identical isotherms. All gangliosides were more than 99\% pure (the highest purity available), as determined by HPLC analysis following the method of Gazzotti et al. [15], and by HPTLC.

The phospholipids used for the experiments have been obtained with a degree of purity higher than $99 \%$. The probes showed in each case a single spot in HPTLC and were used without further purification.

The compounds were spread as (1-5) $\cdot 10^{-3} \mathrm{M}$ chloroform or chloroform/methanol $(2: 1, \mathrm{v} / \mathrm{v})$ solutions. As a standard procedure, monolayer films were rested 5 min before compression to allow sufficient solvent removal (see, for example, Ref. 8), and then compressed by a movable Dynal (polyoxyethylene) barrier at a constant rate of $20 \mathrm{~cm}^{2} / \mathrm{min}$. As the subphase for monolayer preparation, $5 \mathrm{mM}$ triethanolamine (TEA) $/ \mathrm{HCl}$ buffer ( $\mathrm{pH} 7.4$ ) was used or, instead of this, pure deionized water ( $\mathrm{pH}$ 5.6) filtered by a Milli-Q-System without the addition of any ions, or $5 \mathrm{mM}$ phosphate $\left(\mathrm{NaH}_{2} \mathrm{PO}_{4} / \mathrm{Na}_{2} \mathrm{HPO}_{4}\right)$ buffer ( $\left.\mathrm{pH} 7.4\right)$, respectively. The temperature was $20 \pm 0.5^{\circ} \mathrm{C}$. The values reported are the averages of at least 2-5 runs with separately prepared films.

\section{Results and Discresion}

The aim of this study is to determine the potential contribution of the water/lipid interface for gangliosides and different lipids that are common components in biological membranes. For the better understanding of the significant role of gangliosides in the formation and function of biological membranes [16] it is important to know of how far the elongated bulky ganglioside headgroups modify the potential drop across the water/lipid interface with respect to other lipids. 


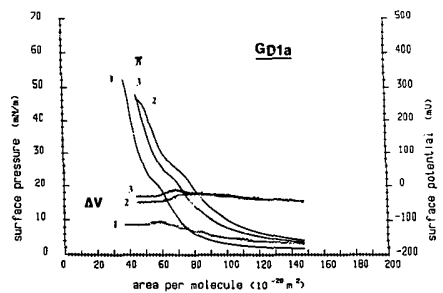

Fig. 1. Surface pressure $(\pi)$-area and surface potential $(\Delta V)$-area isotherms of $\mathrm{G}_{\mathrm{Dl}}$ monolayers at different subphases: Subphase 1: water, pH 5.6. Subphase 2: $5 \mathrm{mM}$ triethanolamine (TEA)/HCl buffer (pH 7.4). Subphase 3: $5 \mathrm{mM}$ phosphate $\left(\mathrm{NaH}_{2} \mathrm{PO}_{4} / \mathrm{Na}_{2} \mathrm{HPO}_{4}\right)$ buffer (pH 7.4).

From three experimental data, i.e. surface pressure $\pi$, surface potential $\Delta V$ and mean molecular area $A$, we have calculated the effective total dipole moment $\Delta_{\perp} \mu$ per molecule as well as the effective local dipole moment $\Delta_{\perp} \mu_{\alpha}$ and the effective local surface potential $\Delta V_{\alpha}$, respectively, of the hydrated polar headgroups at
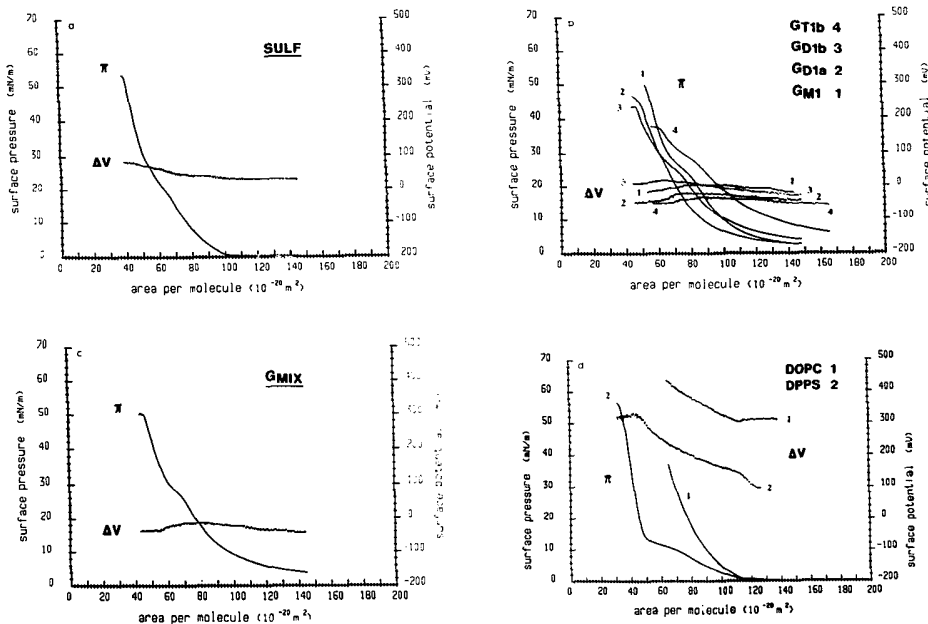

the water/monolayer interface. Since the lateral pressure within biological membranss is expected to be between 20 and $30 \mathrm{mN} / \mathrm{m}[17-20]$ :ve compare the experimental and calculated data at these surface pressures. The measurements were done on pure water as well as on two buffer solutions, namely a $5 \mathrm{mM}$ triethanolamine (TEA/ $\mathrm{HCl}, \mathrm{pH}$ 7.4) and a $5 \mathrm{mM}$ sodium phosphate $(\mathrm{pH}$ 7.4) buffer. The data include charge shielding by counter ions, changes in the dielectric constant of water in the vicinity of the headgroups, and alterations in the location and strength of the dipole sources, depend on the $\mathrm{pH}$ and the salt concentration of the aqueous subphase.

The complete isotherms on these three different subphase conditions are only given for $G_{D 1 a}$ (Fig. 1), but the whole set of data is summarized for all the investigated compounds in Tables I-III. The differences among the components on different subphases are qualitatively quite similar and the main features are comparable. Therefore, the further experimental approaches will be concentrated on monolayers on TEA-buffer solutions ( 5 $\mathrm{mM}, \mathrm{pH} 7.4,20^{\circ} \mathrm{C}$ ). As representative for all compounds the characteristic surface pressure-area and surface potential-area isotherms are given for the sulfatide, different individual gangliosides $\left(\mathrm{G}_{\mathrm{M} 1}, \mathrm{G}_{\mathrm{Dla}}\right.$,

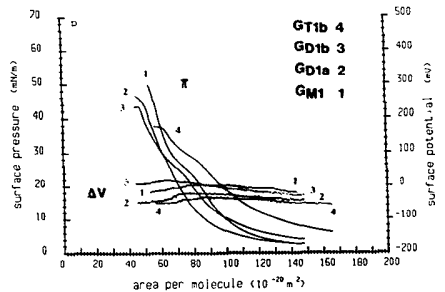

Fig. 2. Surface pressure ( $\pi$ )-area and surface potential ( $\Delta V$ )-area isotherms of (a) Sulf-; (b) $G_{M I}(1)-, G_{D I a}(2), G_{D I b}$ (3)-, $G_{T 1 b}(4)$-; (c) $G_{M i x}$ (d) DOPC (1), DPPS (2)-monolayers. Subphase: $5 \mathrm{mM}$ triethanolamine (TEA)/HCl buffer (pH 7.4), $20 \pm 0.5^{\circ} \mathrm{C} ;$ accuracy $10 \mathrm{mV}$. 


\section{TABLE I}

Surface potentials and effective total dipole moments of close-packed $(\pi=20$ and $30 \mathrm{mN} / \mathrm{m})$ ganglioside, phospholipid and fatty acid monolayers and of their headgroup regions at the air/water interface

The surface pressure $\pi$, the mean molecular area $A$ and the surface potential $\Delta V$ are experimental values. The effective total dipole moment $\Delta_{\perp} \mu$ (Eqn. 2), the effective local dipole moment of the hydrated head group $\Delta_{\perp} \mu_{a}$ (Eqn. 3) and the local surface potential at the water/monolayer interface $\Delta V_{\alpha}$ (Eqn. 2) are calculated based on the dipole values of the terminal $\mathrm{CH}_{3}$-groups of the hydrocarbon chains $\left(\mu_{\mu} \mathrm{CH}_{3}=+0.35\right.$ D) [14]. Subphase: water, pH $5.6,20 \pm 0.5^{\circ} \mathrm{C}$, accuracy $10 \mathrm{mV}\left(1 \mathrm{D}=3.335 \cdot 10^{-30} \mathrm{C} \mathrm{m}\right)$.

\begin{tabular}{lllllll}
\hline $\begin{array}{l}\text { Mono- } \\
\text { layer }\end{array}$ & $\begin{array}{l}\pi \\
(\mathrm{mN} / \mathrm{m})\end{array}$ & $\begin{array}{l}A \\
\left(\mathrm{~nm}^{2}\right)\end{array}$ & $\begin{array}{l}\Delta V \\
(\mathrm{mV})\end{array}$ & $\begin{array}{l}\Delta V_{\alpha} \\
(\mathrm{mV})\end{array}$ & $\begin{array}{l}\Delta_{\perp} \mu \\
(\mathrm{D})\end{array}$ & $\begin{array}{l}\Delta_{\perp} \mu_{\alpha} \\
(\mathrm{D})\end{array}$ \\
\hline $\mathrm{G}_{\mathrm{M1}}$ & 20 & 0.65 & -117 & -527 & -0.199 & -0.899 \\
& 30 & 0.53 & -123 & -623 & -0.171 & -0.871 \\
$\mathrm{G}_{\mathrm{Dia}}$ & 20 & 0.58 & -104 & -559 & -0.160 & -0.860 \\
& 30 & 0.47 & -111 & -679 & -0.137 & -0.837 \\
$\mathrm{G}_{\mathrm{Tlb}}$ & 20 & 0.63 & -146 & -564 & -0.244 & -0.944 \\
& 30 & 0.52 & -142 & -656 & -0.193 & -0.893 \\
$\mathrm{G}_{\text {Mix }}$ & 20 & 0.57 & -105 & -572 & -0.157 & -0.857 \\
& 30 & 0.45 & -106 & -689 & -0.127 & -0.827 \\
DOPC & 20 & 0.78 & +306 & -33 & +0.632 & -0.068 \\
& 30 & 0.70 & +329 & -49 & +0.610 & -0.090 \\
DPPS & 20 & 0.39 & +235 & -451 & +0.240 & -0.460 \\
& 30 & 0.37 & +242 & -476 & +0.236 & -0.464 \\
$\mathrm{C}_{16}$ & 20 & 0.19 & +275 & -413 & +0.140 & -0.210 \\
& 30 & 0.18 & +276 & -446 & +0.134 & -0.217 \\
$\mathrm{C}_{20}$ & 20 & 0.20 & +278 & -386 & +0.146 & -0.204 \\
& 30 & 0.19 & +270 & -434 & +0.135 & -0.215 \\
\hline
\end{tabular}

$\left.\mathrm{G}_{\mathrm{Dlb}}, \mathrm{G}_{\mathrm{Tlb}}\right)$, the ganglioside mixture $\left(\mathrm{G}_{\mathrm{Mix}}\right)$ and two phospholipids (DOPC, DPPS) in Fig. 2 (a-d).

Fig. 2d depicts the surface pressure $(\pi)$-area and surface potential $(\Delta V)$-area curves of DPPS spread on $5 \mathrm{mM}$ TEA $/ \mathrm{HCl}$ buffer. The $\pi$-area curve exhibits under these conditions a two-dimensional phase transition from the liquid-expanded to the liquid-condensed state. This finding differs significantly from that recently published [21]. The differences might be due to the fact that the molecular packing and the phase transition temperature strongly depends on the $\mathrm{pH}$, ionic strength or divalent cations (see references in Ref. 22). This is supported by force-area curves of DPPS spread on pure water or on phosphate buffer (not shown), which are absolutely similar (condensed state, limiting area, collapse pressure) to that shown by Demel and collaborators [21].

The isotherm of DOPC (Fig. 2d) is of the form typically shown by lipids in the liquid-expanded state. The $\pi$-area and $\Delta V$-area curves presented are in agreement with the literature (see, for example, Ref. 23).

As already published by Maggio et al. [8] the surface potential-area curves for gangliosides on $145 \mathrm{mM} \mathrm{NaCl}$
TABLE II

Surface potentials and effective total dipole moments

See text of Table 1. Subphase: $5 \mathrm{mM}$ triethanolamine (TEA) $/ \mathrm{HCl}$ buffer (pH 7.4), $20 \pm 0.5^{\circ} \mathrm{C}$.

\begin{tabular}{|c|c|c|c|c|c|c|}
\hline $\begin{array}{l}\text { Mono- } \\
\text { layer }\end{array}$ & $(\mathrm{mN} / \mathrm{m})$ & $\begin{array}{l}A \\
\left(\mathrm{~nm}^{2}\right)\end{array}$ & $\begin{array}{l}\Delta V \\
(\mathrm{mV})\end{array}$ & $\begin{array}{l}\Delta V_{a} \\
(m V)\end{array}$ & $\begin{array}{l}\Delta_{+}{ }^{\mu} \\
\text { (D) }\end{array}$ & $\begin{array}{l}\Delta_{1} \mu_{a} \\
\text { (D) }\end{array}$ \\
\hline$\overline{\mathrm{G}_{\mathrm{MI}}}$ & $\begin{array}{l}20 \\
30\end{array}$ & $\begin{array}{l}0.84 \\
0.67\end{array}$ & $\begin{array}{r}-2 \\
-17\end{array}$ & $\begin{array}{l}-316 \\
-413\end{array}$ & $\begin{array}{l}-0.005 \\
-0.029\end{array}$ & $\begin{array}{l}-0.705 \\
-0.729\end{array}$ \\
\hline $\mathbf{G}_{\mathrm{Dla}}$ & $\begin{array}{l}20 \\
30\end{array}$ & $\begin{array}{l}0.82 \\
0.64\end{array}$ & $\begin{array}{l}-17 \\
-35\end{array}$ & $\begin{array}{l}-342 \\
-453\end{array}$ & $\begin{array}{l}-0.037 \\
-0.059\end{array}$ & $\begin{array}{l}-0.737 \\
-0.759\end{array}$ \\
\hline $\mathrm{G}_{\text {Dlb }}$ & $\begin{array}{l}20 \\
30\end{array}$ & $\begin{array}{l}0.72 \\
0.62\end{array}$ & $\begin{array}{l}+17 \\
+18\end{array}$ & $\begin{array}{l}-349 \\
-408\end{array}$ & $\begin{array}{l}+0.033 \\
+0.029\end{array}$ & $\begin{array}{l}-0.667 \\
-0.671\end{array}$ \\
\hline $\mathbf{G}_{\mathrm{TIb}}$ & $\begin{array}{l}20 \\
30\end{array}$ & $\begin{array}{l}0.97 \\
0.75\end{array}$ & $\begin{array}{l}-35 \\
-39\end{array}$ & $\begin{array}{l}-307 \\
-391\end{array}$ & $\begin{array}{l}-0.089 \\
-0.078\end{array}$ & $\begin{array}{l}-0.789 \\
-0.778\end{array}$ \\
\hline $\mathbf{G}_{\text {Mix }}$ & $\begin{array}{l}20 \\
30\end{array}$ & $\begin{array}{l}0.77 \\
0.61\end{array}$ & $\begin{array}{l}-15 \\
-24\end{array}$ & $\begin{array}{l}-359 \\
-455\end{array}$ & $\begin{array}{l}-0.030 \\
-0.039\end{array}$ & $\begin{array}{l}-0.730 \\
-0.739\end{array}$ \\
\hline Sulf & $\begin{array}{l}20 \\
30\end{array}$ & $\begin{array}{l}0.63 \\
0.51\end{array}$ & $\begin{array}{l}+56 \\
+69\end{array}$ & $\begin{array}{l}-365 \\
-448\end{array}$ & $\begin{array}{r}+0.093 \\
+0.093\end{array}$ & $\begin{array}{l}-0.607 \\
-0.607\end{array}$ \\
\hline Cer & $\begin{array}{l}20 \\
30\end{array}$ & $\begin{array}{l}0.35 \\
0.34\end{array}$ & $\begin{array}{l}+574 \\
+578\end{array}$ & $\begin{array}{l}-178 \\
-208\end{array}$ & $\begin{array}{l}+0.534 \\
+0.515\end{array}$ & $\begin{array}{l}-0.166 \\
-0.185\end{array}$ \\
\hline DOPC & $\begin{array}{l}20 \\
30\end{array}$ & $\begin{array}{l}0.77 \\
0.69\end{array}$ & $\begin{array}{l}+396 \\
+420\end{array}$ & $\begin{array}{l}+51 \\
+38\end{array}$ & $\begin{array}{r}+0.805 \\
+0.769\end{array}$ & $\begin{array}{l}+0.105 \\
+0.065\end{array}$ \\
\hline DPPE & $\begin{array}{l}20 \\
30\end{array}$ & $\begin{array}{l}0.43 \\
0.41\end{array}$ & $\begin{array}{l}+595 \\
+599\end{array}$ & $\begin{array}{l}-25 \\
-49\end{array}$ & $\begin{array}{r}+0.671 \\
+0.648\end{array}$ & $\begin{array}{l}-0.029 \\
-0.052\end{array}$ \\
\hline DPPS & $\begin{array}{l}20 \\
30\end{array}$ & $\begin{array}{l}0.45 \\
0.42\end{array}$ & $\begin{array}{l}+328 \\
+329\end{array}$ & $\begin{array}{l}-255 \\
-296\end{array}$ & $\begin{array}{l}+0.394 \\
+0.369\end{array}$ & $\begin{array}{l}-0.306 \\
-0.331\end{array}$ \\
\hline DOPA & $\begin{array}{l}20 \\
30\end{array}$ & $\begin{array}{l}0.68 \\
0.61\end{array}$ & $\begin{array}{l}+220 \\
+250\end{array}$ & $\begin{array}{l}-168 \\
-186\end{array}$ & $\begin{array}{l}+0.397 \\
+0.402\end{array}$ & $\begin{array}{l}-0.303 \\
-0.298\end{array}$ \\
\hline DOMA & $\begin{array}{l}20 \\
30\end{array}$ & $\begin{array}{l}0.53 \\
0.50\end{array}$ & $\begin{array}{l}+810 \\
+843\end{array}$ & $\begin{array}{l}+306 \\
+316\end{array}$ & $\begin{array}{l}+1.126 \\
+1.121\end{array}$ & $\begin{array}{l}+0.426 \\
+0.421\end{array}$ \\
\hline $\mathrm{C}_{18}$ & $\begin{array}{l}20 \\
30\end{array}$ & $\begin{array}{l}0.21 \\
0.19\end{array}$ & $\begin{array}{l}+209 \\
+210\end{array}$ & $\begin{array}{l}-434 \\
-477\end{array}$ & $\begin{array}{l}+0.114 \\
+0.107\end{array}$ & $\begin{array}{l}-0.236 \\
-0.234\end{array}$ \\
\hline
\end{tabular}

\section{TABLE III}

Surface potentials and effective total dipole moments

See text of Table I. Subphase: $5 \mathrm{mM}$ phosphate $\left(\mathrm{NaH}_{2} \mathrm{PO}_{4} / \mathrm{Na}_{2}\right.$ $\mathrm{HPO}_{4}$ ) buffer (pH 7.4), $20 \pm 0.5^{\circ} \mathrm{C}$.

\begin{tabular}{lllllll}
\hline $\begin{array}{l}\text { Mono- } \\
\text { layer }\end{array}$ & $\begin{array}{l}\pi \\
(\mathrm{mN} / \mathrm{m})\end{array}$ & $\begin{array}{l}A \\
\left(\mathrm{~nm}^{2}\right)\end{array}$ & $\begin{array}{l}\Delta V \\
(\mathrm{mV})\end{array}$ & $\begin{array}{l}\Delta V_{\mathrm{a}} \\
(\mathrm{mV})\end{array}$ & $\begin{array}{l}\Delta_{+} \mu \\
(\mathrm{D})\end{array}$ & $\begin{array}{l}\Delta_{+} \mu_{\alpha} \\
(\mathrm{D})\end{array}$ \\
\hline $\mathrm{G}_{\mathrm{M}}$ & 20 & 0.75 & +5 & -346 & +0.010 & -0.690 \\
& 30 & 0.60 & -8 & -449 & -0.013 & -0.713 \\
$\mathrm{G}_{\text {Dla }}$ & 20 & 0.71 & -14 & -386 & -0.026 & -0.726 \\
& 30 & 0.56 & -30 & -506 & -0.044 & -0.744 \\
$\mathrm{G}_{\mathrm{Tlb}}$ & 20 & 0.82 & -40 & -364 & -0.086 & -0.786 \\
& 30 & 0.65 & -43 & -452 & -0.074 & -0.774 \\
$\mathrm{G}_{\text {Mix }}$ & 20 & 0.70 & -10 & -386 & -0.019 & -0.719 \\
& 30 & 0.56 & -23 & -495 & -0.034 & -0.734 \\
DOPC & 20 & 0.78 & +404 & +63 & +0.830 & +0.130 \\
& 30 & 0.70 & +431 & +52 & +0.795 & +0.095 \\
DPPS & 20 & 0.44 & +412 & -197 & +0.474 & -0.226 \\
& 30 & 0.41 & +423 & -223 & +0.458 & -0.242
\end{tabular}


at $\mathrm{pH} 5.6$ are flat, whereas the neutral glycosphingolipids exhibit, in part, marked changes in the surface potential-area curves (see also Ref. 24). The negati*ely charged sulfatide shows a less strong increase than the uncharged cerebroside [7]. The physical properties of sulfatide have been extensively studied (for reviews see Refs. 5 and 6). Using differential scanning calorimetry (DSC) it is demonstrated that brain sulfatide forms lamellar-gel and liquid-crystalline phases, and undergoes a broad acyl chain order-disorder transition with a peak maximum at about $50^{\circ} \mathrm{C}$. The presence of a transition shoulder (Fig. 2a) is in contrast to studies of Maggio et al. [10], however, Thompson and Tillack [5], have pointed out that the value of the transition temperature $T_{m}$ depends strongly on the type of counter cation and its concentration.

In gangliosides most of the well-known monolayer isotherms had been measured about 10 years ago [8] with the extraction and purification procedures available to that time. It is noteworthy that, up to this day, in most of the relevant publications these curves are cited, and the calculations of molecular parameters like surface potential/molecule and resultant dipole moments $\Delta_{\perp} \mu$ as well as comparisons to the thermotropic behaviour [10] based on these early measurements. The surface pressure-area isotherms of some individual gangliosides, reported by Maggio et al. [8], besides some differences in molecular area [11-13], exhibited no shoulders ('kinks') at $20^{\circ} \mathrm{C}$. In control experiments using identical subphase conditions (unbuffered 145 $\mathrm{mM} \mathrm{NaCl}, \mathrm{pH}$ 5.6) according to IMaggio et al. [8], we observed quite similar isotherms compared to those shown here on the TEA/HCl buffer subphase.

Probst et al. [11] published similar data showing the disagreement to Maggio et al. [8], and suggested, that the expansion of the monolayers and the abolishing of the phase transition shoulders could be explained by the presence of surface-active contaminants in their gang- lioside solutions, and they duly reported that the shoulders could always be detected by their method.

Using means for eliminating contaminants from the pure gangiioside solutions equally rigorous as described by Gazzotti et al. [15,25], as well as investigating drugs $\left(G_{M 1}, G_{M i x}=\right.$ 'Cronassial'), and working with a monolayer balance of higher sensitivity than that used in former studies, we now point out our findings. It is left to the scientific community to provide furiher experimental evidence for resolution of this continuing disagreement.

With respect to this phase transition, it becomes apparent from listing the results obtained by some different authors that great care must be taken when the occurence of phase transition and transition temperature is postulated [26-33]. Thompson and Tillack [5] have suggested that the differences in the thermotropic behaviour may be due to the existence of metastability in ganglioside micelle systems resulting in a strong structural dependence on the method of preparation and thermal history.

On principle, the ganglioside thermal transitions are related to a cinange from one disordered state to another possibly more disordered state and are not analogous to the gel to liquid-crystalline transitions seen in phospholipids and sphingomyelin [27]. Since the enormous headgroup comprises more than half the bulk of the ganglioside molecule, during monolayer compression the transition observed in the isotherms (Figs. 1, 2b and 2c) might also be caused by rearrangements of the polar headgroups [11]. Bunow and Bunow [28] have suggested that changes in packing efficiency and hydrogen-bonding in the polar headgroup, seen for $G_{M}$. may be reflected in the lower enthalpic peak by differencial scanning calorimetry.

Monolayers of fatty acid $\left(\mathrm{C}_{18}\right)$ as well as of the phospholipids (DOPC and DPPE, representative of the uncharged phospholipid matrix of neuronal mem-
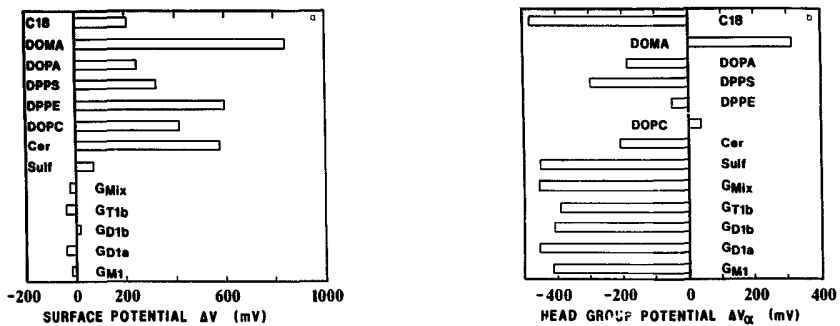

Fig. 3. (a) Surface potential $\Delta V$ of close-packed $(\pi=30 \mathrm{mN} / \mathrm{m})$ monolayers at the air/water interlace. (b) Effective local surface potential $\Delta V_{\pi}$ at the water/monolayer interface (headgroup region) calculated from the data given in Table l1. S $5 \cdot b_{0}$ base: $5 \mathrm{mM} /$ triethanolamine (TEA)/HCl buffer (pH 7.4), $20 \pm 0.5^{\circ} \mathrm{C}$ : accuracy $10 \mathrm{mV}$. 
branes: DPPS and DOPA, representative of the negativeiy charged hospholipids) give rise to positive surface potentials within close-packed monolayers $(\pi=30$ $\mathrm{mN} / \mathrm{m}$ ) ranging from $\Delta V=+210 \mathrm{mV}\left(\mathrm{C}_{18}\right)$ to $\Delta V=$ $+599 \mathrm{mV}$ (DPPE) (Fig. 3a). The total potential of the positively charged quaternary ammonium salt reaches $\Delta V=+843 \mathrm{mV}$ (DOMA). For ceramide, which is the basis for the biosynthesis of gangliosides, a positive potential of $\Delta V=+578 \mathrm{mV}$ is found. The potential of sulfatide carrying one negative charge under the given buffer conditions is still positive $(\Delta V=+69 \mathrm{mV})$. Only the gangliosides, with the exception of $G_{\mathrm{Dtb}}(\Delta V=+18$ $\mathrm{mV}$ ), give rise to negative $\Delta V$-values (Fig. 3a) with the lowest one for $\mathrm{G}_{\mathrm{TIb}}(\Delta V=-39 \mathrm{mV})$. It should be noted that only potential differences with respect to the uncovered water surface are measurable with:

$\Delta V=V_{\text {monolayet }}-V_{\text {air } / \text { water }}$

The pure and uncovered air/water interface is already polarized by spontaneous orientation of the water molecules, and the potential drop across the air/water interface is expected to be negative $(-100$ to $-200 \mathrm{mV})$ [14]. Therefore, after the potential of the water surface is taken into account, the sulfatide as well as all gangliosides give rise to negative potential jumps within close-packed monolayers at the air/water interface. Among the gangliosides no striking differences exist between head groups carrying one or three charges. This result is remarkable and will be discussed later. The surface potential $\Delta V$ and the effective total dipole moment $\Delta_{\perp} \mu$ are connected via the Helmholtz equation

$\Delta_{\perp} \mu=\epsilon_{0} \cdot \Delta V \cdot A$

The air/water interface splits up into two interfaces by the formation of a monolayer: the water/monolayer and the monolayer/air interface. It is appropriate to assume that the dipole fields of both interfaces superpose additively [34], i.e. the total dipole moment $\Delta_{\perp} \mu$ (Fig. 4a) is the sum of the local dipole moments, $\Delta_{\perp} \mu_{\alpha}$ (water/monolayer) and $\mu_{\perp}^{\omega}$ (monolayer/air)

$\Delta_{\perp} \mu=\Delta_{1} \mu_{\alpha}+{ }_{\perp} \mu^{\omega}$

The dipole contribution of ${ }_{\perp} \mu^{\omega}$ of close-packed hydrocarbon chains in contact to air was recently determined experimentally [14] to be ${ }_{\perp} \mu^{\omega}=+0.35 \mathrm{D}$, directed from the monolayer $(-)$ to the air $(+)$. The dipole moment contribution of the terminal $\mathrm{CH}_{3}$ group used in previous papers (see, for example, Ref. 35) ranged from $-0.3 \mathrm{D}$ to at least $+0.3 \mathrm{D}$ and were obtained by calculations. This is in contrast to our value. On principle, the literature values reported so far are based on theoretical calculations and differ even in the sign.

In the close-packed state $(\pi \geqq 20 \mathrm{mN} / \mathrm{m})$ the monolayers of the given lipids resemble each other in the structure of the hydrophobic region: the hydrocarbon chains have a high degree of positional order $[36,37]$ with no or only slight chain tilts. In the glycolipids used in this study, ceramide consisting of the sphingosine moiety with the fatty acid linked to it, gives the hydrophobic properties to the amphiphilic molecules. Sphingosine is generally an unbranched $C_{18}$ or $C_{20}$ hydrocarbon [26] with a primary hydroxyl on carbon 1 , an amino group on carbon 2, and a secondary hydroxyl on carbon 3. A trans double bond connects carbon 4 and 5. The most common fatty acids found in amide linkage to the sphingosine range from $C_{16}$ to $C_{28}$, but brain gangliosides contain mainly stearic acid [5]. It was previously reported by Fidelio et al. [26] that the influence of the hydrocarbon portion heterogeneity on monolayer properties as well as on thermotropic behaviour of micellar dispersions is relatively small compared to the effect of the sugar headgroup. The number of $\mathrm{CH}_{2}$-groups of the hydrocarbon chain that separate the two monolayer interfaces does not contribute to the total effective dipole moment $\Delta_{\perp} \mu[14,38]$. Therefore, we have to assume for a further discussion that the dipole contribution per one chain end at the monolayer/air interface is the same for all of these lipid monolayers.

Before the value given above is applied to the lipid/air interface of ganglioside monolayers, two structural differences between the packing of the hydrocarbon chains of closepacked fatty acids and gangliosides have to be mentioned. In gangliosides an interesting feature of the hydrophobic portion is the variability in chain length of the fatty acid relative to sphingosine portion in ceramide having two unequal chain lengths (generally the difference is up to 4 catbons) [16]. Supposing the amino sphingosine base is $\mathbf{C}_{18}$, this behaves like a fatty acid of 14 or 15 carbons. To estimate the influence of different chains lengths on the dipole contribution of the monolayer/air interface, the effective total dipole moment of a mixed monolayer of fatty acids, $\mathrm{C}_{16}: \mathrm{C}_{20}$, in the molar ratio of $1: 1$ has been compared to the value of the pure layers. The effect of different chain lengths within one monolayer on the local dipole moment of the monolayer/air interface is less than $10 \%$ since the terminal chain order is slightly reduced. A further structural difference concerns the tilt angle of the hydrocarbon chains: the tilt angle of closepacked fatty acids on water is close to zero, whereas the chains of gangliosides are slightly tilted. For arachidic acid on water it is known that the tilt angle changes continueously if the monolayer is compressed from 2 $\mathrm{mN} / \mathrm{m}$ to $25 \mathrm{mN} / \mathrm{m}$ on a counter ion free aqueous subphase [39]. Again, the dipole moment changes less than $10 \%$. Further calculations are based on the value ${ }_{\perp} \mu^{\mathrm{CH}_{3}}=+0.35 \mathrm{D}$ by being aware of its small error bar.

The local dipole moment per head group $\Delta_{\perp} \mu_{\alpha}$ is an effective quantity. It should not be related to the intrin- 
sic dipole moment of the headgroup but includes dipole compensation by the hydration shell as well as charge shielding by counter ions. Therefore, the dipole contribution even of charged head groups to the total dipole moment per molecule does not exceed the contribution from the unpolar monolayer/air interface. It should be noted that for extended headgroups, the relative position of the charge located in the headgroup with respect to the surrounding hydration shell has an effect on the potential drop [14]. The local dipole moment increases, if the accessibility of the charge by water molecules is reduced, e.g., by methylation. In fact the data of the ammonium-containing phospholipid headgroup ethanolamine (-PE: $\left.\Delta_{\perp} \mu_{\alpha}=-0.05 \mathrm{D}\right)$ and the methylated analogue choline (-PC: $\Delta_{\perp} \mu_{\alpha}=+0.07 \mathrm{D}$ ) exhibit this effect. The effective local dipole moments of the investigated headgroups are summarized in Fig. $4 \mathrm{~b}$. With the exception of DOPC and the positively charged headgroup of DOMA $(+0.42 \mathrm{D})$ the headgroups have negative local dipole moments. The local dipole moment of the hydrated carboxyl group of $\mathrm{C}_{18}$ is negative $(-0.23 \mathrm{D})$ and directed from the monolayer $(-)$ to the water $(+)$. Glycosphingolipids have even more negative values ranging from $-0.61 \mathrm{D}$ (Sulf) to $-0.78 \mathrm{D}\left(\mathrm{G}_{\mathrm{TIh}}\right)$. The influence of the number of negative charges per ganglioside headgroup is negligibly small. These findings agree with observations from conductance studies measuring the electrostatic potential of gangliosides $\left(G_{M 1}, G_{D l a}, G_{T 1 b}\right)$ within planar bilayers [40]. As shown in the Tables I-III, the second and third sialosyl group adds a component to the dipole moment, which is of very small magnitude and, likewise in contrast to Maggio et al. $[4,8]$, having no positive air end (with the exception of the second sialosyl group of $\mathbf{G}_{\mathrm{DIb}}$ ). These authors reported the headgroup components were calculated by subtracting a calculated $\psi_{0}$ potential according to Gouy-Chapman, and surprisingly the sec- ond and third sialosyl group adds a positive component to the overall dipole moment. This fact has not been explained convincingly.

We did not follow the usual procedure of subtracting a Gouy-Chapmann $\psi_{0}$ potential since our results show $\left(G_{M 1}\right.$ in comparison with $G_{D 1 a}$ and $\left.G_{T l b}\right)$ that such an approach can be misleading, when the charge density is not clearly defined and the charges are not located at the interfacial plane separating the hydrocarbon moiety from the aqueous subphase. Therefore, we limit ourself to give the apparent dipole moments and use them to calculate the potential drop across the water/lipid interface as presented in Fig. 3b. Only the apparent potential drop across the headgroup plane is significant for the understanding of the biological function of gangliosides.

Only recently, several authors investigated the electrokinetic and electrostatic peculiarities of Lilayers (vesicles, planar membranes) containing gangliosides to predict the potential of ganglioside interfaces whose charges are located deeper into the aqueous phase $[40-42]$. These groups assumed that the ganglioside headgroups project $2.5 \mathrm{~nm}$ from the bilayer, and calculated all fixed charges are in a plane $1 \mathbf{~ n m}$ from the bilayer surface. This approach is actually acceptable only for $G_{M 1}$, but not for the divalent $G_{D_{1 a}}$, and the trivalent $\mathbf{G}_{\mathrm{Tlb}}$.

By means of our monolayer studies the effective local interfacial potential at the water/monolayer interface, $\Delta V_{\alpha}$, is recalculated from the dipole data (Eqns. 2 and 3). With the subphase conditions tested, the local surface potentials at the water/monolayer interface, especially the values of the glycolipid headgroups and the $\mathrm{COO}^{-}$group of fatty acids, reach up to minus several hundred millivolts, which is unexpectedly large (Fig. 3b). It is worth emphasizing the influence of such highly negative potentials especially of gangliosides, with respect to
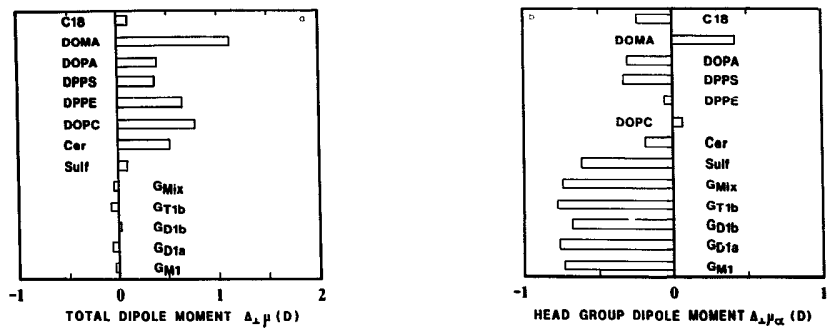

Fig. 4. (a) Effective total dipole moment $\Delta_{\perp} \mu$ of close-packed ( $\pi=30 \mathrm{mN} / \mathrm{m}$ ) monolayers at the air/water interface. (b) Effective local dipole moment $\Delta_{\perp} \mu_{\alpha}$ at the water/monolayer interface (head group region) calculated from the data given in Table II. Subphase: 5 mM triethanolamine (TEA)/HCl buffer (pH 7.4), $20 \pm 0.5^{\circ} \mathrm{C}$; accuracy $10 \mathrm{mV}$, 
maintaining a high proton and cation over-concentration near the surface of neuronal membranes.

Depending on the direction of the effective local dipole moments of the headgroups, especially in gangliosides, the headgroup potential is sufficiently large, for instance, to influence conformational changes in membrane proteins (see also Ref. 43). With regard to the $\Delta V_{\alpha}$ data, an effective local headgroup potential difference of more than $100 \mathrm{mV}$ at different lateral surface pressures (e.g., $\mathrm{G}_{\mathrm{Dla}}$ : $-679 \mathrm{mV}$ at $\pi=30 \mathrm{mN} / \mathrm{m}$ versus $-559 \mathrm{mV}$ at $\pi=20 \mathrm{mN} / \mathrm{m}$ ) as well as a difference of more than $200 \mathrm{mV}$ between DPPS $(-476 \mathrm{mV}$ at $\pi=30$ $\mathrm{mN} / \mathrm{m}$ ) and $\mathrm{G}_{\mathrm{Dla}}$ in membranes is not negligible.

In summary, we have given the sign and the magnitude of the potential drop across the water/lipid interface for different ganglioside, phospholipid and sulfatide monolayers. The apparent dipole monent per ganglioside headgroup differs from other lipids. The most remarkable result is that among gangliosides the size and the number of charges do not cause large changes in the potential drop. Our potential data imply that the main purpose of nature to vary in biomembranes the number of charges per ganglioside headgroup, e.g., from one to three, is not to change the apparent potential drop across the headgroup region.

The headgroup potential of the pure ganglioside monolayers reaches minus several hundred millivolts which can influence the electrostatic properties of neuronal bilayer surfaces strongly [43]. Since the potential and dipole data gained here on monolayers can be directly applied to biomembrane and vesicles systems, the aim of further studies should be to investigate the influence of ionic strength and changes in temperature on these physicochemical properties of pure as well as of binary mixed monolayer films as a basis for processes occurring within cell membranes.

\section{References}

1 Rahmann, H., Rösner, H. and Breer, H. (1976) J. Theor. Biol. 57, 231-237.

2 Wieraszko, A. and Seifert, W. (1986) Brain Res. 371, 305-313.

3 Rahmann, H. (1987) in Gangliosides and Modulation of Neuronal Functions, NATO ASI Series, H7 (Rahmann, H., ed.), pp. 501-521, Springer Verlag, Berlin, Heidelberg.

4 Maggio, B., Cumar, F.A. and Caputto, R. (1981) Biochim. Biophys. Acta 650, 69-87.

5 Thompson, T.E. and Tillack. T.W. (1985) Annu. Rev. Biophys. Biophys. Chem. 14, 361-386.

6 Curatolo. W. (1987) Biochim. Biophys. Acta 906, 111-136.

7 Quinn, P.J. and Sherman. W.R. (1971) Biochim. Biophys. Acta 233, 734-752.

8 Maggio, B., Cumar, F.A. and Caputto, R. (1978) Biochem. J. 171, 559-565.

9 Mazgio, B., Cumar, F.A. and Caputto, R. (1980) Biochem. J. 189, $435-440$.
10 Maggio, B., Sturtevant. J.M. and Yu, R.K. (1987) Biochim. Biophys. Acta 901, 173-182.

11 Probst, W., Möbius, D. and Rahmann, H. (1984) Cell. Mol. Neurobiol. 4, 157-176.

12 Beitinger. H., Probst, W., Möbius, D. and Rahmann, H. (1987) J. Biochem. 102, 963-966.

13 Schifferer, F. Beitinger, H., Rahmann, H, and Möbius, D. (1988) FEBS Lett. 233, 158-162.

14 Vogel, V. and Möbius, D. (1988) Thin Solid Films 159, 73-81

15 Gazzotti, G., Sonnino, S. and Ghidoni, R. (1985) J. Chromatogr. 348, 371-378.

16 Grant, C.W.M. (1987) in Gangliosides and Modulation of Neuronal Functions, NATO ASI Series, H7 (Rahmann, H., ed.), pp. 119-138, Springer/Berlin, Heidelberg.

17 Marcelja, S. (1974) Biochim. Biophys. Acta 367, 165-176.

18 Blume. A. (1979) Biochim. Biophys. Acta 557, 32-44.

19 Fulford, A.J.C. and Pell, W.E. (1980) Biochim. Biophys. Acta 598, 237-246.

20 Israelachvili, J.N., Marcelja, S. and Horn, R.G. (1980) Q. Rev. Biophys. 13, 121-200.

21 Demel, R.A., Paltauf, F. and Hauser, H. (1987) Biochemistry 26. 8659-8665.

22 Sacré, M.M. and Tocanne, J.F. (1977) Chem. Phys. Lipids 18, 334-354.

23 Paltauf, F., Hauser, H. and Phillips, M.C. (1971) Biochim. Biophys. Acta 249, 539-547.

24 Oldani, D., Hauser, H., Nichols, B.W. and Phillips, M.C. (1975) Biochim. Biophys. Acta 382, 1-9.

25 Gazzotti, G., Sonnino, S., Ghidoni, R., Kirschner, G. and Tettamanti, G. (1984) J. Neurosci. Res. 12, 179-192.

26 Fidelio, G.D., Maggio, B. and Cumar, F.A. (1986) Biochim. Biophys. Acta 854, 231-239.

27 Curatolo, W., Small, D.k1. and Shipley, G.G. (1977) Biochim. Biophys. Acta 468, 11-20.

28 Bunow, M.R. and Bunow, B. (1979) Biophys. J. 27, 325-337.

29 Bach, D., Miller, I.R. and sela, B.-A. (1982) biochim. Biophys. Acta 686, 233-239.

30 Uchida, T., Nagai, Y., Kawasaki, Y. and Wakayama, N. (1981) Biochemistry 20, 162-169.

31 Sillerud, L.O., Schafer, D.E., Yu. R.K. and Königsberg. W.O. (1979) J. Biol. Chem. 254, 10876-10880.

32 Hinz, H.-J., Körner, O. and Nicolau, C. (1981) Biochim. Biophys. Acta 643, 557-571.

33 Kojima, H., Hanada-Yoshikawa, K., Katagiri, A. and Tamai, Y. (1988) J. Biochem. 103, 126-131.

34 Davies, J.T. and Rideal, E.K. (1963) Interfacial Phenomena, pp. 75-92, Academic Press, New York.

35 Bernet, M.K., Jarvis, N.L. and Zisman, W.A. (1964) J. Phys. Chem. 68, 3520-3529.

36 Möhwald, H. (1988) Thin Solid Films 159, 1-15.

37 Dutta, P., Peng, J.B., Lin, B., Ketterson, J.B., Prakash, M., Georgopoulos, P. and Ehrlich, S. (1987) Phys. Rev. Lett. 58, 2228-2231.

38 Bernett, M.K. and Zisman, W.A. (1963) J. Phys. Chem. 67, 1534-1540.

39 Helm, C.A. (1988) Thesis, Tech. Univ, Munich, Munich.

40 McDaniel, R.V., Sharp, K., Brouks, D., McLaughlin, A.C., Winiski, A.P., Cafiso, D. and Mclaughlin, S. (1986) Biophys. J. 49, 741-752.

41 McDaniel, R.V., McLaughlin, A., Winiski, A.P., Eisenberg, M. and McLaughlin, S. (1984) Biochemistry 23, 4618-4624.

42 Winiski, A.P., Eisenberg, M., Langner, M. and McLaughlin, S. (1988) Biochemistry 27, 386-392.

43 Seelig, J., MacDonald, P.M. and Scherer, P.G. (1987) Biochemistry $26,7535-7541$. 